\title{
Medición del coeficiente de transferencia del oxígeno en aguas residuales
}

Measurement of the coefficient of oxygen in wastewater transfer

Recibido: julio 12 de 2018 | Revisado: agosto 11 de 2018 | Aceptado: setiembre 18 de 2018

\author{
Pedro Córdova Mendoza \\ Dante Calderón Huamaní ${ }^{1}$ \\ George Argota Pérez ${ }^{2}$
}

\section{RESUMEN}

La acumulación de materia orgánica en los cuerpos de aguas receptores condiciona limitaciones sobre el valor de uso siendo la concentración de oxígeno disuelto, uno de los factores a estimar para su biodegradación. El propósito del estudio fue medir el coeficiente de transferencia del oxígeno en aguas residuales. Se determinó la demanda bioquímica de oxígeno y la demanda química de oxígeno durante cinco horarios $(0,20,45,70$ y 90 minutos). Los resultados fueron: $\mathrm{DBO}_{5} ; 5554,33 \pm 4,041$ (O minutos), 3807,0 $\pm 4,0$ (20); $2616,33 \pm 7,505$ (45); 1466,33 $\pm 5,686(70)$ y $579,67 \pm 4,041$ (90). En el caso de la DQO fueron: 8095,33 $\pm 4,041(0)$; 6954 $\pm 7,505$ (20); $4791,0 \pm 4,582$ (45); $2342,67 \pm 4,509$ (70) y $1786,0 \pm 3,605(90)$. Se encontró diferencias estadísticamente significativas $(\mathrm{p}<0,05)$ entre los valores por tiempo para cada parámetro. Al compararse los valores hallados con los recomendados, superaron el estándar de calidad ambiental permisible para las aguas $\left(\mathrm{DBO}_{5}=5,0 \mathrm{mg} \cdot \mathrm{L}^{-1}\right.$; $\mathrm{DQO}=20,0$ mg. $\left.\mathrm{L}^{-1}\right)$. Se estableció la relación: $f=\mathrm{DBO}_{5} /$ DQO donde el cociente fue: 0,544 y se concluyó que, las aguas residuales presentaron condiciones de biodegradabilidad pudiendo ser utilizado un tipo de tratamiento específico.

Palabras clave: coeficiente de transferencia del oxígeno, biodegradación, materia orgánica, agua residual

\section{Abstract}

The accumulation of organic matter in receptive bodies of water conditions limitations on the value of use being the concentration of dissolved oxygen one of the factors to estimate for its biodegradation. The purpose of the study was to measure the oxygen transfer coefficient in wastewater. The biochemical oxygen demand and the chemical oxygen demand were determined during five schedules $(0,20,45$, 70 and 90 minutes). The results were: BOD5; $5554.33 \pm 4.041(\mathrm{O}$ minutes), $3807.0 \pm 4.0(20) ; 2616.33 \pm 7.505$ (45); $1466.33 \pm 5.686$ (70) and $579.67 \pm 4.041$ (90). In the case of the COD were: 8095.33 $\pm 4.041(0) ; 6954 \pm 7.505(20) ; 4791.0 \pm 4.582(45) ; 2342.67 \pm 4.509$ (70) and $1786.0 \pm 3.605$ (90). Statistically significant differences were found $(\mathrm{p}<0.05)$ between the values per time for each parameter. When the found values were compared with the recommended ones, they exceeded the environmental quality standard allowed for water $($ BOD5 $=5.0 \mathrm{mg} . \mathrm{L}-1, \mathrm{COD}=20.0 \mathrm{mg} . \mathrm{L}-1)$. The relationship was established as: $\mathrm{f}=\mathrm{BOD} 5 / \mathrm{COD}$ where the quotient was: 0.544 and it was concluded that the wastewater presented biodegradability conditions and a specific type of treatment could be used.

Key words: oxygen transfer coefficient, biodegradation, organic matter, waste water 


\section{Introducción}

La degradación ambiental sobre la calidad de las aguas está condicionando su limitación en diversas regiones del planeta debido a, la persistencia no solo de contaminantes disueltos y/o acumulados (Wang \& Zang, 2014; Hezhong et al., 2015), además, en la variabilidad de parámetros físico-químicos y la concentración sobre materia orgánica que en algunos casos ya es irreversible en término de costo ambiental para el manejo del agua (He et al., 2014; Argota, Argota \& Iannacone, 2016).

Por lo general, las actividades de pesquisas se realizan cuando las condiciones del cuerpo acuático receptor comienza a degradarse; y donde cualquier información sobre su calidad debe cumplirse de forma anticipada en términos preventivos (Mouquet et al., 2015; Petchey et al., 2015) pudiendo contribuir quizás, hacia la eficiencia holística (integración económica y ambientales) de las plantas de tratamientos para aguas residuales y así, asegurar la disminución de elementos no deseados cuya finalidad entonces sería, la sostenibilidad sobre el uso del agua durante largo periodo de tiempo (Färe $e t$ al., 2014; Fuentes et al., 2015; Lu, Du \& Huang, 2017).

El oxígeno disuelto es una de los factores reguladores ambientales y cuando es limitante, existe baja tasa de reducción-oxidación (Kim et al., 2013) como acumulación de la materia orgánica, incluyendo la biodegradable, pues la biomasa microbiana aerobia al ser directamente proporcional a la concentración de oxígeno disuelto (Park \& Noguera, 2004), entonces resultaría imposible, eliminarse aquella materia orgánica en exceso no requerida y donde Guo et al., (2013) indican que, en estas condiciones se produce eutrofización de los sistemas acuáticos. El propósito del estudio fue medir el coeficiente de transferencia del oxígeno en aguas residuales.

\section{Materiales y métodos}

El estudio se realizó en la laguna de oxidación perteneciente al distrito de Guadalupe, provincia de Ica, departamento de Ica. En marzo del 2018, se muestreó aguas residuales durante cinco horarios $(0,20,45,70$ y 90 minutos) donde se determinó la demanda bioquímica de oxígeno $\left(\mathrm{DBO}_{5}\right)$ y la demanda química de oxígeno (DQO). Luego se calculó el coeficiente de transformación (f) según la siguiente expresión:

$$
f=\mathrm{DBO}_{5} / \mathrm{DQO}
$$

Para la caracterización del tipo de agua residual se siguió lo indicado en la Tabla 1.

Tabla 1

Coeficiente de transformación del agua residual

\begin{tabular}{c|c|c}
\hline $\boldsymbol{f}$ & tipo de agua residual & biodegradabilidad \\
\hline$>0,7$ & urbanas puras & muy biodegradables \\
$0,2-0,7$ & industriales & biodegradables \\
$<0,2$ & & no biodegradables \\
\hline
\end{tabular}

Fuente: Gil, 2005 
Para el análisis de los datos se utilizó el programa profesional Statgraphics Centurion XVI.II y la normalidad de los datos se realizó mediante la prueba de Shapiro W.

Para la comparación entre las medias $\left(\mathrm{DBO}_{5}, \mathrm{DQO}\right)$ según la tasa horaria se usó el análisis de varianza y analizó la homogeneidad de grupos con el procedimiento múltiple de Bonferroni. Los resultados fueron comparados con los valores de estándares de calidad ambiental de agua: Decreto Supremo $\mathrm{N}^{\circ}$ 004-2017-MINAM

Tabla 2

Medición de parámetros / agua residual

\begin{tabular}{c|c|c}
\hline Tiempo $(\mathrm{h})$ & $\begin{array}{c}\text { DBO }_{5} \\
\left(\mathrm{mg.L}^{-1}\right)\end{array}$ & $\begin{array}{c}\text { DQO } \\
\left(\mathrm{mg.L}^{-1}\right)\end{array}$ \\
\hline 0 & $5554,33 \pm 4,041$ & $8095,33 \pm 4,041$ \\
20 & $3807,0 \pm 4,0$ & $6954 \pm 7,505$ \\
45 & $2616,33 \pm 7,505$ & $4791,0 \pm 4,582$ \\
70 & $1466,33 \pm 5,686$ & $2342,67 \pm 4,509$ \\
90 & $579,67 \pm 4,041$ & $1786,0 \pm 3,605$ \\
\hline Promedio & $2608,21 \pm 1708,26$ & $4793,93 \pm 2561,72$ \\
Referencia & 5,0 & 20,0 \\
\hline
\end{tabular}

Tabla 3

Análisis de la varianza

\begin{tabular}{|c|c|c|c|c|c|c|}
\hline Parámetro & Fuente de variación & $\begin{array}{l}\text { Suma de } \\
\text { Cuadrados }\end{array}$ & $\begin{array}{l}\text { Grados de } \\
\text { libertad }\end{array}$ & $\begin{array}{c}\text { Cuadrado } \\
\text { Medio }\end{array}$ & $\begin{array}{l}\text { Coeficiente } \\
\text { Fisher }\end{array}$ & Valor $\mathbf{P}$ \\
\hline \multirow{3}{*}{$\mathrm{DBO} 5$} & Entre grupos & $4,60438 \mathrm{E} 7$ & 4 & $1,15109 \mathrm{E} 7$ & 419087,79 & 0,0000 \\
\hline & Intra grupos & 274,667 & 10 & 27,4667 & & \\
\hline & Total (Corr,) & $4,60441 \mathrm{E} 7$ & 14 & & & \\
\hline \multicolumn{7}{|c|}{ Grupos Homogéneos } \\
\hline $\mathrm{DBO}_{5} 90$ & $\mathrm{a}$ & & & & & \\
\hline $\mathrm{DBO}_{5} 70$ & $b$ & & & & & \\
\hline $\mathrm{DBO}_{5} 45$ & c & & & & & \\
\hline $\mathrm{DBO}_{5} 20$ & d & & & & & \\
\hline $\mathrm{DBO}_{5} 0$ & $\mathrm{e}$ & & & & & \\
\hline \multirow{3}{*}{ DQO } & Entre grupos & $1,38385 \mathrm{E} 8$ & 5 & $2,76771 \mathrm{E} 7$ & 835884,14 & 0,0000 \\
\hline & Intra grupos & 397,333 & 12 & 33,1111 & & \\
\hline & Total (Corr,) & $1,38386 \mathrm{E} 8$ & 17 & & & \\
\hline
\end{tabular}




\begin{tabular}{lc}
\hline & Grupos Homogéneos \\
DQO 90 & a \\
DQO 70 & b \\
DQO 45 & c \\
DQO 45 & d \\
DQO 20 & e \\
\hline
\end{tabular}

Al determinarse el coeficiente de transformación se halló un cociente de: 0,544 lo cual indicó que las muestras de aguas fueron biodegradables y por tanto, es posible su tratamiento.

$$
\begin{gathered}
f=2608,21 \mathrm{mg} \cdot \mathrm{L}^{-1} / 4793,93 \mathrm{mg} \cdot \mathrm{L}^{-1} \\
=0,544
\end{gathered}
$$

Muñoz et al., (2012) mencionaron que la contaminación de los ríos en México, es un problema ambiental ya que las fuentes de materia orgánica son diversas y entre ellas se encuentran las actividades agrícolas, industriales y las aguas residuales generadas en las zonas urbanas y rurales. Como resultado de su estudio los valores de $\mathrm{DBO}_{5}$ fueron entre 0,74 y 169,93 mg. $\mathrm{L}^{-1}$ mientras que, la DQO estuvo entre 2,16 y 440,05 mg. $\mathrm{L}^{-1}$, respectivamente. En este estudio, los valores hallados comparativamente fueron superiores.

Otra investigación realizada por Jie, Kang \& Anderson (2018), utilizando la $\mathrm{DBO}_{5}$ como parámetro de influyente predictivo mencionó que, la concentración umbral fue de $87,5 \mathrm{mg} . \mathrm{L}^{-1}$ siendo este valor muy inferior al encontrado en el presente estudio. Los autores refirieron que el control sobre este parámetro es crítico para proporcionar confiabilidad en las operaciones a nivel de planta de tratamiento.

En una investigación sobre la eliminación de la demanda química de oxí- geno por electrodeposición de aguas residuales mediante purificado y polímero adsorbentes de nanotubos de carbono funcionales se encontró que antes de la aplicación tecnológica, la concentración fue de 1094 mg.L-1 (Bankole et al., 2017) que se halla por debajo al promedio de nuestro estudio lo cual demuestra, que se consumen altas concentraciones de oxígeno para oxidar (degradar) la materia orgánica.

En otro estudio para la eliminación de la demanda química de oxígeno en aguas residuales utilizando bentonita recubierta de quitosano, la concentración fue de $1348,00 \mathrm{mg} . \mathrm{L}^{-1}$ igualmente por debajo al promedio de nuestro estudio (Ligaray et al., 2018). Estos resultados comparados pudieron indicar que, las aguas residuales presentaron materia orgánica (cargas contaminantes) de forma constante.

Finalmente, la relación entre el $\mathrm{DBO}_{5}$ / DQO no solamente, es usada para aguas residuales, también lo es, en el análisis de lixiviados para su calidad y donde algunos parámetros pueden ser incorporados como es el pH y la temperatura (Bhatt et al., 2017).

Se concluyó que, las aguas residuales presentaron condiciones de biodegradabilidad donde puede aplicarse, algún tipo de tratamiento específico. 


\section{Referencias}

Argota, P.G., Argota, C.H. \& Iannacone, O.J. (2016). Costo ambiental sostenible relativo a la variabilidad físico-química de las aguas sobre la disponibilidad de metales en el ecosistema San Juan, Santiago de Cuba, Cuba. The Biologist (Lima); 14(2), 219-232. http://dx.doi. org/:10.24039/rtb201614299

Bhatt, A.H., Karanjekar, R.V., Altouqi, S., Sattler, M.L., Sahadat, H.M.D. Chen, V.P. (2017). Estimating landfill leachate BOD and COD based on rainfall, ambient temperature, and waste composition: Exploration of a MARS statistical approach. Environmental Technology \& Innovation; 8, 1-16. http://dx. doi.org/10.1016/j.eti.2017.03.003

Bankole, M.T., Abdulkareem, S.A., Tijani, J.O., Ochigbo, S.S., Afolabi, A.S. \& Roos, W.D. (2017). Chemical oxygen demand removal from electroplating wastewater by purified and polymer functionalized Carbon nanotubes adsorbents, Water Resources and Industry; http://dx.doi.org/10.1016/j. wri.2017.07.001

Färe, R., Grosskopf, S. \& Pasurka, C.A. (2014). Potential gains from trading bad outputs: The case of us electric power plants. Resource and Energy Economics; 36, 99-112. http://dx.doi.org/:10.1016/.reseneeco.2013.11.004

Fuentes, R., Torregrosa, T. \& Ballenilla, E. (2015). Conditional order-m efficiency of wastewater treatment plants: The role of environmental factors, Water; 7, 55035524. https://doi.org/10.3390/ w7 105503

Guo, J., Zhang, L., Chen, W., Ma.F., Liu, H. \& Tian, Y. (2013). The regulation and control strategies of a sequencing batch reactor for simultaneous nitrification and denitrification at different temperatures. Bioresour Technol; 133 5967. http://dx.doi.org/:10.1016/j. biortech.2013.01.026

He, J., Zhang, H., Zhang, H., Guo, X., Song, M., Zhang, J. \& Li, X. (2014). Ecological risk and economic loss estimation of heavy metals pollution in the Beijiang River. Ecological Chemistry and Engineering; 21:189-199. https://doi. org/:10.2478/eces-2014-0015

Hezhong, Y., Wei, P., Zhengjie, Z. Ying, W., Qifang, G. \& Shuqing, A. (2015). Ecological risk assessment of heavy metals in sediments of riverine wetland, Huaihe river watershed, China. Ecol Chem Eng $S ;$ 22(2), 231-242. https://doi. org/:10.1515/eces-2015-0013

Jie, Z.J., Kang, L. \& Anderson, P.R. (2018). Predicting influent biochemical oxygen demand: Balancing energy demand and risk management. Water Research; 128, 304313. https://doi.org/10.1016/j. watres.2017.10.053 
Kim, Y,M., Park, H., Cho, K.H. \& Park, J.M. (2013). Long term assessment of factors affecting nitrifying bacteria communities and $\mathrm{N}$-removal in a full-scale biological process treating high strength hazardous wastewater. Bioresour Technol; 134, 180189. http://dx.doi.org/:10.1016/j. biortec.2013.02.036

Lu, B., Du, X. \& Huang, S. (2017). The economic and environmental implications of wastewater management policy in China: From the LCA perspective. Journal of Cleaner Production, 142, 3544-3557. http://dx.doi.org/10.1016\%2Fj. jclepro.2016.10.113

Mouquet, N., Lagadeuc, Y., Devictor, V., Doyen, L., Duputié, A. \& et al. (2015). Predictive ecology in a changing world. J Appl Ecol; 52, 1293-1310. http://dx.doi. org/10.1111/1365-2664.12482

Muñoz, N.H., Suárez, S.J., Vera, R.A., Orozco, F.S., Batlle, S.O. et al. (2012). Demanda bioquímica de oxígeno y población en la sub- cuenca del río Zahuapan, Tlazcala, México. Rev. Int. Contam. Ambie; 28(1), 27-38. http://www.scielo. org. mx/pdf/rica/v28n1/v28n1a3. pdf

Park, H.D. \& Noguera, D.R. (2004). Evaluating the effect of dissolved oxygen on ammonia oxidizing bacterial communities in activated sludge. Water Res; 38, 32753286. http://dxdoi.org/:10.1016/j. watres 200404.047

Petchey, O.L., Pontarp, M., Massie, T.M., Kéfi, S., Ozgul, A. \& et al. (2015). The ecological forecast horizon and examples of its uses and determinants. Ecol Lett; 18, 597-611. https://doi.org/10.1111/ ele. 12443

Wang, X. \& Zang, S. (2014). Distribution characteristics and ecological risk assessment of toxic heavy metals and metalloid in surface water of lakes in Daqing Heilonjiang Province, China. Ecotoxicology; 23, 609617. https://doi.org/10.1007/ s10646-014-1177-y 